\title{
Optimized sympathetic cooling of atomic mixtures via fast adiabatic strategies
}

\author{
Stephen Choi, ${ }^{1}$ Roberto Onofrio, ${ }^{2,1,3}$ and Bala Sundaram ${ }^{1}$ \\ ${ }^{1}$ Department of Physics, University of Massachusetts, Boston, MA 02125, USA \\ ${ }^{2}$ Dipartimento di Fisica "Galileo Galilei", Università di Padova, Via Marzolo 8, Padova 35131, Italy \\ ${ }^{3}$ ITAMP, Harvard-Smithsonian Center for Astrophysics, 60 Garden Street, Cambridge, MA 02138, USA
}

(Dated: November 6, 2018)

\begin{abstract}
We discuss fast frictionless cooling techniques in the framework of sympathetic cooling of cold atomic mixtures. It is argued that optimal cooling of an atomic species - in which the deepest quantum degeneracy regime is achieved - may be obtained by means of sympathetic cooling with another species whose trapping frequency is dynamically changed to maintain constancy of the Lewis-Riesenfeld adiabatic invariant. Advantages and limitations of this cooling strategy are discussed, with particular regard to the possibility of cooling Fermi gases to a deeper degenerate regime.
\end{abstract}

PACS numbers: 37.10.De, 37.10.Gh, 37.10.Vz

The continuous progress in achieving low temperatures has opened up new research directions in atomic physics, most notably the formation and study of degenerate Bose and Fermi gases. For the latter, most of the interesting physics, such as novel superfluid states or ordered phases, is conditional on the achievement of very low degeneracy factors $T / T_{\mathrm{F}}$, now limited to above $\simeq 10^{-2}$ [1, 2]. In most of the experiments the Fermi gas is in thermal contact with a Bose gas cooled with the usual evaporative cooling strategy and having a much larger heat capacity than the Fermi gas, resulting in the so-called sympathetic cooling (see [3 5] for earlier demonstrations). However, both the loss of atoms intrinsic to evaporative cooling and the strong dependence on temperature of the heat

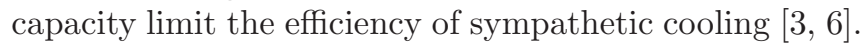

If the main goal of the experiments is to achieve the deepest Fermi degeneracy, an alternative procedure can be envisaged with the use of species-selective traps in which the Bose gas alone is kept as close as possible to the nondegenerate regime, as proposed for bichromatic optical traps in [7], and for light-assisted magnetic traps in [8]. Recently, species-selective light-assisted magnetic traps have been successfully implemented to sympathetically cool Bose-Bose mixtures such as ${ }^{41} \mathrm{~K}-{ }^{87} \mathrm{Rb}[9]$ and ${ }^{87} \mathrm{Rb}^{-174} \mathrm{Yb}$ [10], and these experimental techniques could be naturally extended to Fermi-Bose mixtures. However, the ultimate, optimal ideal coolant would be a gas that retains the maximum value of its heat capacity, both because it does not enter the degenerate regime, and also because the number of atoms is not diminished during the cooling stage. These two features are common to a new class of cooling techniques known under the name of frictionless (or, less rigorously - as seen in later considerations - fast adiabatic) cooling. This method was pioneered in atomic physics in [11], and experimentally implemented for fast decompression of ${ }^{87} \mathrm{Rb}$ atoms both in the nondegenerate [12] and degenerate 13 regimes, and for demonstrating fast atomic transport [14].

It is the purpose of this Rapid Communication to ex- plore the extent to which frictionless cooling techniques may be useful in sympathetic cooling of Fermi gases, while also discussing their practical limitations. An important motivating factor is that an usually undesired feature of frictionless cooling, i.e. the fact that the atomic cloud does not increase its phase space density and therefore its degeneracy, is instead a crucial asset from the perspective of maintaining the gas in the nondegenerate regime and making it an ideal coolant.

Fast frictionless cooling [11] is based on the use of an adiabatic invariant for harmonically trapped atoms of mass $m$ - hereafter assumed in the nondegenerate regime and therefore with negligible interactions. Following Lewis and Riesenfeld [15, 16], we consider the operator $\hat{I}(t)=\hat{\pi}^{2} / 2 m+m \omega_{0}^{2} \hat{q}^{2} /\left(2 b^{2}\right)$ where $\hat{\pi}=b \hat{p}-m \dot{b} \hat{q}$ is the momentum operator conjugate to the operator $\hat{q} / b$. The parameter $\omega_{0}$ can be chosen as the initial frequency, and $b(t)$ is a time-dependent frequency scaling factor which must satisfy, in order for $\hat{I}(t)$ to be an invariant operator, the Ermakov equation $\ddot{b}(t)+\omega(t)^{2} b=$ $\omega_{0}^{2} / b^{3}$. This can be solved by imposing boundary conditions on $b(t)$ and its first and second time derivatives, if we assume both a targeted final trapping frequency $\omega_{\mathrm{f}}$ and a total time duration for the adiabatic protocol $t_{\mathrm{f}}$ [11], thus obtaining a well-defined Ermakov trajectory for the requested time-dependent trapping frequency as $\omega^{2}(t)=\omega_{0}^{2} / b^{4}(t)-\ddot{b}(t) / b(t)$.

In our analysis, we compare three decompression strategies for an harmonically trapped atomic cloud as shown in Fig. 1 where, for concreteness, we assume the initial trap frequency $\omega_{0}=2 \pi \times 250 \mathrm{~Hz}$ and the final angular frequency $\omega_{\mathrm{f}}=2 \pi \times 2.5 \mathrm{~Hz}, \omega_{0} / \omega_{\mathrm{f}}=10^{2}$. Two of the trajectories for $\omega^{2}(t)$ (continuous and dashed lines) are solutions of the Ermakov equation and therefore leave the Lewis-Riesenfeld operator invariant in time. The main difference in the two trajectories is the absence (continuous) or the presence (dashed) of a stage in which the square frequency becomes negative. As shown in [11], the presence of an anti-trapping stage appears for small 


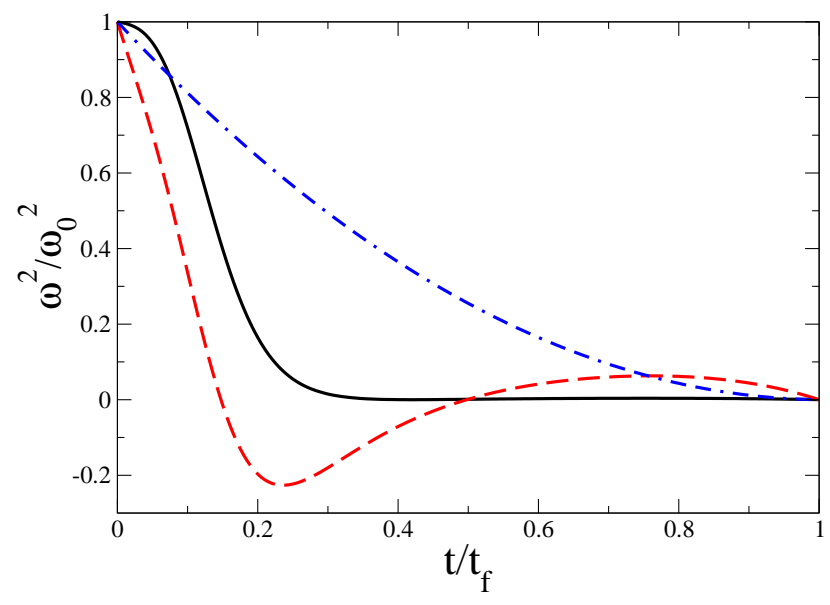

FIG. 1: (Color online) Comparison of various decompression strategies as seen from the square of the trapping frequency, normalized to its initial value, versus time normalized to the overall duration of the decompression. The curves here and in the following figures indicate an Ermakov trajectory with positive square frequency lasting $25 \mathrm{~ms}$ (black, continuous line), a fast Ermakov trajectory of total duration of $6 \mathrm{~ms}$ (red, dashed line), and a linear ramp down of the trapping frequency approximating a conventional slow adiabatic strategy (blue, dot-dashed line) with duration of $400 \mathrm{~ms}$. Notice in particular, that $\omega(t)^{2} / \omega_{0}^{2}<0$ in a finite interval in the 6 ms case, leading to an anti-trapping stage, which occurs for all choices of $t_{\mathrm{f}}<25 \mathrm{~ms}$.

targeted times $t_{\mathrm{f}}$. Physically, the anti-trapping stage enables the wave function to spread out faster so as to reach the final target width within given $t_{\mathrm{f}}$. It is interesting to note that the Ermakov equation typically produces a trajectory where, after anti-trapping, the trap frequency first increases to a value above $\omega_{\mathrm{f}}$ before finally relaxing down to $\omega_{\mathrm{f}}$. This may be interpreted as a way to control the excess momentum directed away from the origin attained during the anti-trapping stage. Experimentally, an anti-trapping stage can be obtained by properly modulating the power of blue-detuned beams, as discussed in [7]. For comparison, the third trajectory shown (dotdashed line) is a naive decompression strategy based on a linear ramp down of the frequency to its targeted final value. Its overall duration is chosen as $t_{\mathrm{f}}=T_{\max }$, where $T_{\max }=2 \pi / \omega_{\mathrm{f}}$ is the longest harmonic oscillator period during the evolution. In this way we compare two Ermakov-related cooling strategies to a strategy which is adiabatic in the usual sense of the word, i.e. with parameters changing slowly with respect to the intrinsic timescale set by the harmonic oscillator frequencies.

We have numerically solved the Schrödinger equation for the time-dependent harmonic oscillator evolving under the $\omega(t)$ solution of the Ermakov equation. By comparing the solution with the solution obtained from the effective Gaussian dynamics formalism [17] it was found that the wave function, if prepared initially as a Gaus-
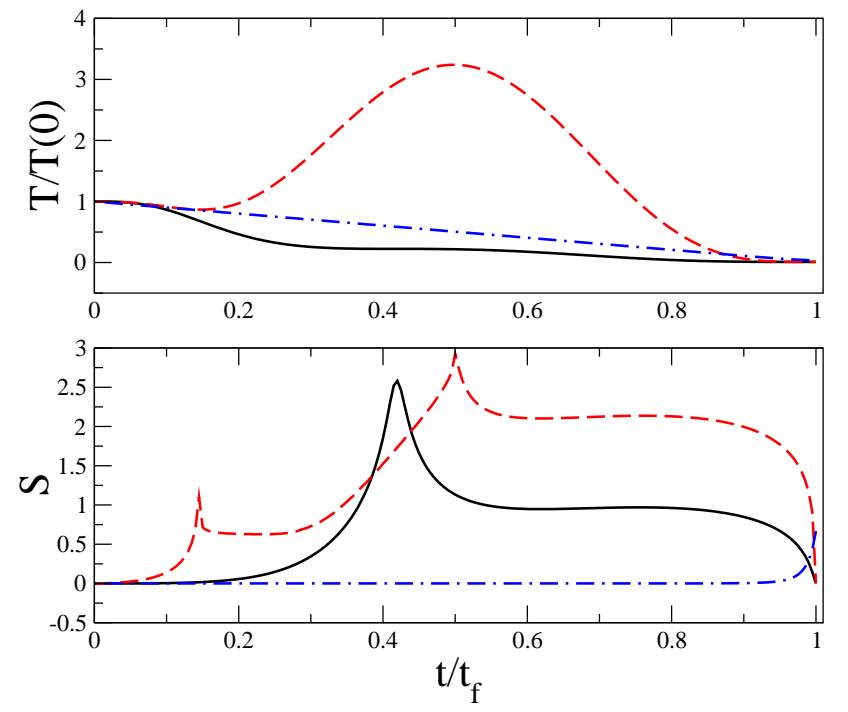

FIG. 2: (Color online) Thermodynamics of the decompression. Temperature scaled to initial temperature (top) and Shannon entropy (bottom) versus time for the three cooling strategies described in Fig. 1. In the case of the decompression strategy corresponding to $t_{\mathrm{f}}=6 \mathrm{~ms}$ the temperature exceeds the initial temperature in a time interval related with the time interval in which the square frequency becomes negative, while in the case of $t_{\mathrm{f}}=25 \mathrm{~ms}$ the temperature behavior is decreasing monotonically. The threshold situation in which the maximum temperature during the anti-trapping stage equals the initial temperature is achieved for $t_{\mathrm{f}} \approx 11$ ms.

sian, retains its Gaussian form throughout the evolution, which proves useful in characterizing the temperature of the cloud. The velocity distribution is routinely used to estimate experimentally the temperature of ultracold atomic gases. In our case, the Gaussian state can be used to represent an ensemble of atoms with a normally distributed position and momentum such that, by assuming Maxwell-Boltzmann distribution, the variance of the velocity distribution is $\sigma_{v}^{2}=k_{B} T / m$ where $k_{B}$ is the Boltzmann constant and $T$ is the temperature of the atomic cloud. At the same time, the use of the variance of the velocity distribution, or equivalently the momentum variance, allows us to monitor the energy population probabilities during the cooling procedure. This is simply a reflection of the fact that a larger momentum variance implies the occupation of a large number of excited momentum states. This complements Ref. [11] where the total energy is used as an indicator of temperature. The time evolution of the temperature is shown for the three cases in the top plot of Fig. 2. In addition, the entropy $S(t)$ is evaluated based on its Shannon definition, $S(t)=-\sum_{n}\left|c_{n}(t)\right|^{2} \log \left|c_{n}(t)\right|^{2}$ where the instantaneous eigenstate basis $\left|\psi_{n}(t)\right\rangle$ corresponding to the harmonic trap frequency $\omega(t)$ is used in the projection of the timeevolved wave function. Although $S(t)$ is the Shannon 
entropy instead of thermodynamic entropy, an adiabatic process would keep $c_{n}(t)$ constant in time, meaning the entropy would remain constant.

In the case of the frictionless strategy with an antitrapping stage the basis for the inverted harmonic oscillator [18] was used $(\hbar=m=1)$ :

$$
\begin{aligned}
\psi_{1}(x, t)= & N \exp \left(\frac{i \omega x^{2}}{2}-\frac{\omega t}{2}+\frac{i \epsilon}{2 \omega} e^{-2 \omega t}\right) \times \\
& \cos \left(e^{-\omega t} \sqrt{\epsilon} x\right) \\
\psi_{2}(x, t)= & \exp \left(\frac{i \omega x^{2}}{2}+i k e^{-\omega t} x-\frac{\omega t}{2}+\frac{i k^{2}}{4 \omega} e^{-2 \omega t}\right)
\end{aligned}
$$

where $\psi_{1}(x, t)$ corresponds to the solution for which the particle is confined to an expanding box with a moving boundary condition. $N$ is the normalization constant and the discrete energy eigenvalues are $E_{n}=e^{-2 \omega t} \epsilon$ where $\epsilon=\left(n+\frac{1}{2}\right)^{2} \pi^{2} / L_{0}^{2}$ with $n$ an integer and $L_{0}$ is the width of the wave function as it first enters the inverted potential. $\psi_{2}(x, t)$ corresponds to the plane wave solution with $k$ being the wave number, and since the plane wave solution cannot be normalized, there is no normalization constant. It was found that, as the anti-trapping stage lasts for extremely short duration typically around $0.25 t_{\mathrm{f}}$, there is not enough time for our wave functions to couple to the free-particle plane wave solution of $\psi_{2}(x, t)$. Therefore our wave functions project entirely onto eigenstates $\psi_{1}(x, t)$. The results of this entropy calculation are shown in the bottom panel of Fig. 2.

Two general features are worth commenting on. First, for the two cases corresponding to the conservation of the Lewis-Riesenfeld invariant, entropy is clearly not constant, which implies that adiabaticity does not hold during the cooling process. The maximum entropy appears at the point where the trap is shallowest while in cases where an anti-trapping region exists, the spikes in entropy are where the curvature of the trap changes its sign. However, the final entropy exactly equals the initial one for these cases, as if a real adiabatic process has taken place. This is in stark contrast to the linear ramp down decompression where the entropy is constant throughout the evolution except as we approach the final time. This increase is expected as the decreasing frequency leads to stricter conditions for true adiabaticity, indicating a need for much longer timescales for the ramp-down.

Second, the difference between the linear decrease and the Ermakov constructs is also clear in the temperature dynamics. Higher intermediate temperatures correspond to the presence of an anti-trapping stage, which in turn appears in the Ermakov solution for shorter $t_{\mathrm{f}}$. We note that in general the presence of a stage in which the temperature of the cloud may become much higher than the initial cloud temperature is problematic. Since atomic traps have a finite potential depth, a rise of the cloud temperature will induce losses of the most energetic atoms [19]. From our standpoint, this leads to a practical limita-

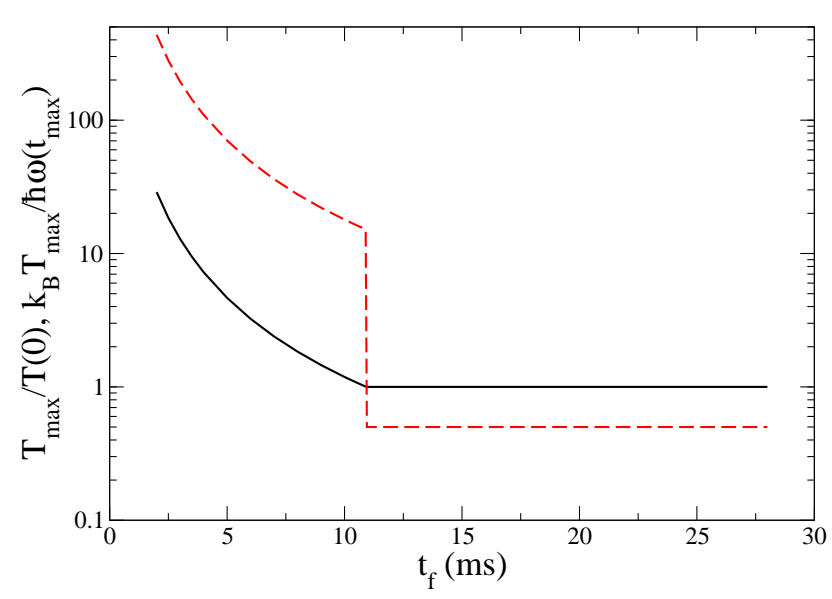

FIG. 3: (Color online) Dependence of the maximum temperature attained versus the duration of the cooling strategy $t_{\mathrm{f}}$ for the targeted temperature decrease $T\left(t_{\mathrm{f}}\right) / T(0)=10^{-2}$. Solid line: maximum achieved temperature scaled to the initial temperature. Dashed line: maximum temperature expressed in units of quanta of the harmonic oscillator with the instantaneous frequency corresponding to the time at which the maximum temperature occurs. For $t_{\mathrm{f}}$ larger than $\approx 11$ $\mathrm{ms}$, the initial temperature is also the maximum temperature. For shorter $t_{\mathrm{f}}$, substantially higher temperatures occur after the anti-trapping region as seen in the top plot in Fig. 2.

tion on the minimum possible time for the cooling strategy, with a natural tradeoff also determined by the lifetime of the atoms in the trap. The final temperature for the linear ramp case is slightly higher than that for the other two cases. However, if the linear ramp occurred on the same time scales as the other two fast adiabatic methods, the final temperature would be much higher [20]. In order to quantify this potential bottleneck of the cooling protocol, we show in Fig. 3 the maximum achieved temperature, i.e. the peak value of the temperature in Fig. 2, scaled to the initial temperature, versus the time duration of the cooling procedure. In the same figure we also show the maximum achieved temperature in units of quanta of the corresponding instantaneous harmonic oscillator $k_{B} T_{\max } / \hbar \omega\left(t_{\max }\right)$. This figure of merit is useful to assess trap losses in the case of a finite trap depth. The results show, that for $t_{\mathrm{f}}$ shorter than approximately $11 \mathrm{~ms}$, the maximum temperature of the atoms becomes comparable to the energy of the $15^{\text {th }}$ excited state of the instantaneous harmonic trap, and quickly increases for shorter $t_{\mathrm{f}}$. Nevertheless, it appears that optimal cooling with minimal trap loss on timescales comparable to the oscillation period of the trap is possible as long as excessively short cooling times are avoided. This is clear from the temperature curve where for $t_{\mathrm{f}}>11 \mathrm{~ms}$, the initial temperature is also the highest temperature indicating stability in the dynamics as the trap is made more shallow. By contrast, at shorter times, substantial heating resulting from the anti-trapping region would make the fric- 
tionless strategy difficult to implement. The maximum heating due to anti-trapping was found to occur typically at $t \simeq t_{\mathrm{f}} / 2$, the time when the trap changes its curvature at the end of anti-trapping. The sudden "jump" occurs at around $t_{\mathrm{f}} \approx 11 \mathrm{~ms}$, since for this case the maximum heating due to anti-trapping has the same magnitude as the initial temperature. We emphasize that, as anti-trapping occurs for all $t_{\mathrm{f}}<25 \mathrm{~ms}$ in our example, the presence of an anti-trapping stage alone is not a sufficient condition for heating of the atoms to temperatures greater than the initial temperature.

A different issue arises when the spatial overlap of the two clouds is taken into account, as the coolant species considerably increases its size during its decompression. As discussed in [11], the position variance is directly related to the frequency scaling factor $b$ as $\sigma_{x}^{2}=\hbar(n+1 / 2) b^{2} /\left(m \omega_{0}\right)$. For the two adiabatic invariant strategies, the temporal variation of the position variance is independent of $t_{\mathrm{f}}$ as expected from the fact that the position variance is proportional to $b(t)$. Since $\sigma_{x}^{2}\left(t_{\mathrm{f}}\right) / \sigma_{x}^{2}(0)=b^{2}\left(t_{\mathrm{f}}\right) / b^{2}(0)=\omega_{0} / \omega_{\mathrm{f}}=10^{2}$ as a result of the boundary condition for $b(t)$, it is conceivable that if a large $b\left(t_{\mathrm{f}}\right)$ is targeted, the spread of the atomic cloud of the coolant will result in a small overlap with the cloud to be sympathetically cooled. The linear ramp down result also exhibits a large broadening expected of an adiabatic process that relaxes the trap frequency by a factor of $10^{2}$. The overlap issue seems to be crucial in various experiments (see for instance 21] for the effect of gravitational sagging on a large mass ratio Fermi-Bose mixture), and its effect on species-selective traps has been discussed in detail in [22]. We note that, in practice, the issue of spatial overlap is less severe than expected, since for accidental reasons the fermionic isotope of the alkali primarily used in the experiments, ${ }^{6} \mathrm{Li}$, is lighter than the bosonic species used as coolants, ${ }^{23} \mathrm{Na},{ }^{87} \mathrm{Rb}$, and bosonic isotopes of $\mathrm{Yb}$. Therefore the initial overlap between the two species sees the fermionic species more spread out than the bosonic species. The decompression of the bosonic species alone in general allows for a better spatial overlap at intermediate times, but this also provides a limit on the maximum allowed decompression before the spatial overlap decreases again. It is also worth to point out that, especially for very short cooling times, a possible issue arises with the sympathetic equilibration rate between the two species, depending on the interspecies elastic scattering rate. Although this issue should be studied in detail for each specific atomic mixture, in general this is less relevant than the spatial overlap, and can be circumvented by using magnetic or optical Feshbach resonances to boost the interspecies elastic scattering length as in the case of the ${ }^{6} \mathrm{Li}^{87} \mathrm{Rb}$ Fermi-Bose mixture 23 25.

In conclusion, we have discussed the possibility of achieving deep Fermi degeneracy via sympathetic cooling using a frictionless strategy for the coolant species.
The identified advantages in this setting are the maximal heat capacity retained by the coolant due to the conservation of the number of atoms and the preservation of its phase space density in the nondegenerate regime where the specific heat retains its Dulong-Petit value. At the same time we have also identified two important limitations, a cooling stage in which the temperature rises significantly allowing for trap losses if the potential energy depth is not large enough, and the spreading of the cooling cloud which will reduce the spatial overlap with the fermionic cloud. The coolant does not need necessarily to be a Bose gas, as it is kept in the non-degenerate regime during the entire process, thus providing further flexibility in any possible experimental implementation of this scheme.

[1] W. Ketterle and M. W. Zwierlein, Rivista del Nuovo Cimento 31, 247 (2008).

[2] S. Giorgini, L. P. Pitaevskii, S. Stringari, Rev. Mod. Phys. 80, 1215 (2008).

[3] A. G. Truscott, K. E. Strecker, W. I. McAlexander, G. B. Partridge, and R. G. Hulet, Science 291, 2570 (2001).

[4] F. Schreck, L. Khaykovich, K. L. Corwin, G. Ferrari, T. Bourdel, J. Cubizolles, and C. Salomon, Phys. Rev. Lett. 87, 080403 (2001).

[5] Z. Hadzibabic, C. A. Stan, K. Dieckmann, S. Gupta, M. W. Zwierlein, A. Görlitz, and W. Ketterle, Phys. Rev. Lett. 88, 160401 (2002).

[6] C. Presilla and R. Onofrio, Phys. Rev. Lett. 90, 030404 (2003).

[7] R. Onofrio and C. Presilla, Phys. Rev. Lett. 89, 100401 (2002).

[8] M. A. Brown-Hayes and R. Onofrio, Phys. Rev. A 70, 063614 (2004).

[9] J. Catani, G. Barontini, G. Lamporesi, F. Rabatti, G. Thalhammer, F. Minardi, S. Stringari, and M. Inguscio, Phys. Rev. Lett. 103, 140401 (2009).

[10] F. Baumer, F. Münchow, A. Görlitz, S. E. Maxwell, P. S. Julienne, and E. Tiesinga, Phys. Rev. A 83, 040702(R) (2011).

[11] X. Chen, A. Ruschhaupt, S. Schmidt, A. del Campo, D. Guéry-Odelin, and J. G. Muga, Phys. Rev. Lett. 104, 063002 (2010).

[12] J.-F. Schaff, X.-L. Song, P. Vignolo, and G. Labeyrie, Phys. Rev. A 82, 033430 (2010).

[13] J.-F. Schaff, X.-L. Song, P. Capuzzi, P. Vignolo, and G. Labeyrie, EPL 93, 23001 (2011).

[14] E. Torrontegui, S. Ibàñez, Xi Chen, A. Ruschhaupt, D. Guéry-Odelin, and J. G. Muga, Phys. Rev. A 83, 013415 (2011).

[15] H. R. Lewis, Phys. Rev. Lett. 18, 510 (1967).

[16] H. R. Lewis and W. B. Riesenfeld, J. Math. Phys. (N.Y.) 10, 1458 (1969).

[17] A. K. Pattanayak and W. C. Schieve, Phys. Rev. A 46, 1821 (1992).

[18] C. Yuce, A. Kilic, and A. Coruh, Phys. Scr. 74, 114 (2006).

[19] B. Ramachandhran, S. G. Bhongale, and H. Pu, Phys. 
Rev. A 83, 033607 (2011).

[20] We have also explored the robustness of the cooling procedure with respect to small deviations from the targeted frequency trajectory, by superimposing to it small perturbations, both of sinusoidal and broadband noise character. In the typical case of a sinusoidal perturbation with amplitude $1 \%$ of the instantaneous frequency and period equal to $10^{-2} t_{\mathrm{f}}$ we obtain $T_{\mathrm{f}}$ differing by $0.1 \%$ with respect to the unperturbed one.

[21] A. H. Hansen, A. Khramov, W. H. Dowd, A. O. Jamison, V. V. Ivanov, and S. Gupta, Phys. Rev. A 84, 011606(R)
(2011).

[22] M. Brown-Hayes, Q. Wei, C. Presilla, and R. Onofrio, Phys. Rev. A 78, 013617 (2008).

[23] C. Silber, S. Günther, C. Marzok, B. Deh, Ph. W. Courteille, and C. Zimmermann, Phys. Rev. Lett. 95, 170408 (2005).

[24] B. Deh, C. Marzok, C. Zimmermann, and Ph. W. Courteille, Phys. Rev. A 77, 010701(R) (2008).

[25] Z. Li, S. Singh, T. V. Tscherbul, and K. W. Madison, Phys. Rev. A 78, 022710 (2008). 